\section{Tick-Borne Relapsing Fever Caused by Borrelia persica in Traveler to Central Asia, 2019}

\author{
Veronika Muigg, ${ }^{1}$ Helena M.B. Seth-Smith, ${ }^{1}$ \\ Daniel Goldenberger, Adrian Egli, Beatrice Nickel, \\ Roland Dürig, Esther Kuenzli, Vladimira Hinic, ${ }^{2}$ \\ Andreas Neumayr ${ }^{2}$
}

Author affiliations: Swiss Tropical and Public Health Institute, Basel, Switzerland; and University of Basel, Basel (V. Muigg, B. Nickel, E. Kuenzli, A. Neumayr); University Hospital Basel, Basel (H.M.B. Seth-Smith, D. Goldenberger, A. Egli, V. Hinic); University of Basel, Basel (H.M.B. Seth-Smith, A. Egli);

Maihofpraxis, Luzern, Switzerland (R. Dürig)

\section{DOI: https://doi.org/10.3201/eid2604.191771}

We report a case of tick-borne relapsing fever caused by Borrelia persica in a traveler returning to Switzerland from central Asia. After the disease was diagnosed by blood smear microscopy, the causative Borrelia species was confirmed by shotgun metagenomics sequencing. PCR and sequencing techniques provide highly sensitive diagnostic tools superior to microscopy.

$\mathrm{W}^{\mathrm{s}}$ e report a case of tick-borne relapsing fever (TBRF) in a 21-year-old male tourist who returned from Kyrgyzstan in July 2019 after having traveled for 5 months to Mexico, Taiwan, and central Asia (Uzbekistan, Tajikistan, and Kyrgyzstan). While in Tajikistan, he experienced acute fever of $39.5^{\circ} \mathrm{C}$, chills, and generalized aches on June 11, which lasted 3 days. He experienced identical episodes around June 17 and 25.

After returning to Switzerland, he sought care on June 28 from his general practitioner, who referred him to the regional hospital, where malaria test results were negative. After the patient experienced 2 more episodes of fever (July 2 and 14), the general practitioner referred him to a tropical medicine specialist on July 15. Anamnesis revealed that the patient had consumed unpasteurized milk and had been bitten by insects nightly while trekking in Tajikistan. Other than fever of $38.5^{\circ} \mathrm{C}$ and pain on palpation of the liver, physical examination revealed no pathologic findings. Abdominal sonography showed a borderline enlarged spleen but was otherwise unremarkable. Chest radiography indicated no abnormalities. Laboratory results are shown in the Appendix (https://wwwnc.cdc.gov/EID/article/26/4/ 19-1771-App1.pdf).

\footnotetext{
${ }^{1}$ These first authors contributed equally to this article.
}

${ }^{2}$ These senior authors contributed equally to this article.
Detection of spirochetes in blood films (Figure) confirmed the diagnosis of a relapsing fever borreliosis, already suspected from the classical presentation of recurrent fever episodes separated by asymptomatic intervals of $\approx 1$ week. Shortly after starting doxycycline, the patient experienced a self-limiting crisis with chills and fever of $41^{\circ} \mathrm{C}$, which we interpret as Jarisch-Herxheimer reaction. Subsequently, the patient's condition rapidly improved.

To determine the Borrelia species, we performed $16 S$ rRNA gene sequencing from the blood sample. Analysis of traces of capillary-sequenced amplified DNA after broad-range 16S rRNA gene PCR (660bp), performed by using RipSeqMixed (Pathogenomix, https://www.pathogenomix.com), could not differentiate between Borrelia recurrentis and B. persica within the 5 ' end of the 16S rRNA gene. Therefore, we used a short-read shotgun metagenomic sequencing approach on DNA on an Illumina NextSeq500 platform (https:/ / www.illumina.com).

Of the 7.8 million sequencing reads, $692(0.009 \%$ of the sequence data) mapped (by CLC Genomics Workbench v.12.0.3 [QIAGEN, https://www.qiagen.com] with a length fraction of 0.8 and a similarity fraction of 0.95 ) to a derived database of Borrelia genomes comprising reference genomes of $B$. recurrentis (GenBank accession nos. CP000999-CP001000), B. persica (Assembly accession AYOT), B. duttonii (Assembly accession AZIT), B. hispanica (Assembly accession AYOU), and B. crocidurae (GenBank accession no. LN609267). The top hit was to B. persica, with 684 $(98.8 \%)$ mapped reads, followed by B. duttoni with 6 reads and $B$. recurrentis with 2 reads. Across the $B$. persica reference genome, reads from the isolate in this case mapped across the whole genome, representing sections of 101 of the 245 assembly scaffolds. We submitted the Borrelia reads to the European Nucleotide Archive (https://www.ebi.ac.uk/ena) under project PRJEB35490. We did not submit the 16S RNA gene sequence to GenBank because of the low quality of the sequence (multiple undetermined nucleotides).

These results strongly suggest that $B$. persica was the infectious agent of TBRF. Pending microscopic confirmation, we ordered several serologic studies, including assays to detect antibodies against the Borrelia species that cause Lyme disease and against rickettsial pathogens (Appendix Table 1). Whether the mildly elevated serologic titer for spotted fever Rickettsia resulted from cross-reactivity or co-infection with a tick-borne Rickettsia remains unclear.

TBRF occurs in temperate and tropical countries and is caused by several species of Borrelia maintained in enzootic cycles in which small mammals serve as 

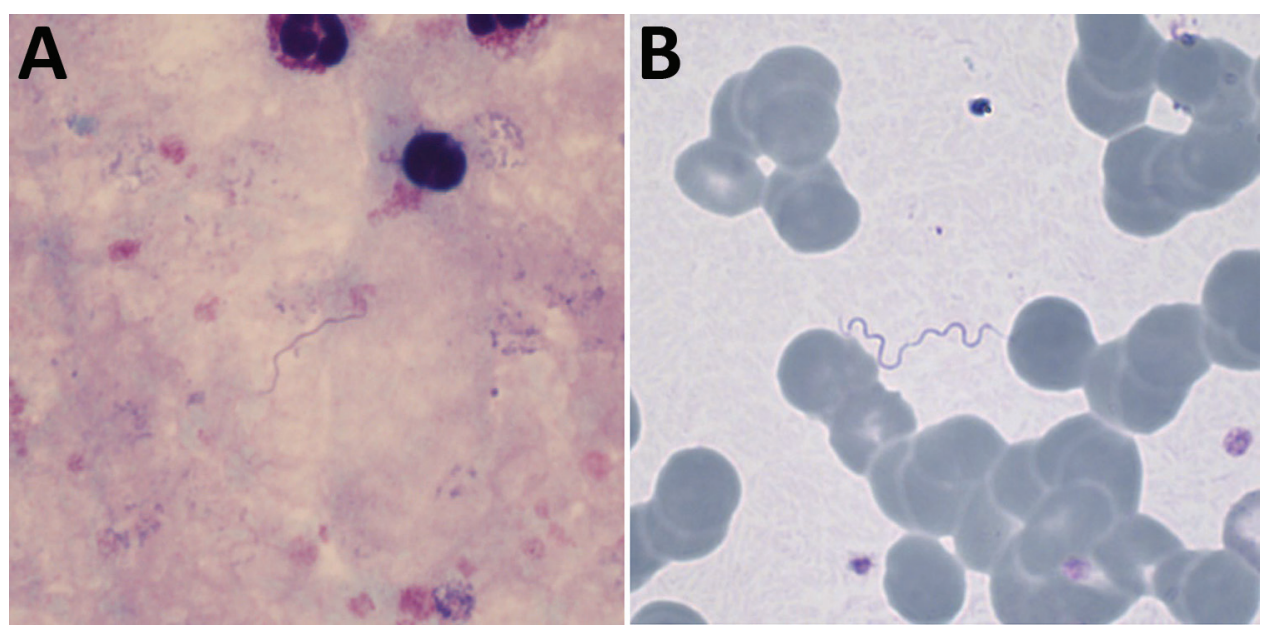

Figure. Giemsa-stained thick (A) and thin (B) blood films, demonstrating extracellular spirochetes. Original magnifications $\times 1,000$.

animal reservoirs and Ornithodoros soft ticks as vectors. Humans are accidental hosts (except for B. duttonii in Africa, which seems strictly limited to humans with no identified animal reservoir), usually exposed to tick bites when sleeping in rustic cabins or caves (1). The disease is characterized by recurrent fever episodes separated by afebrile periods and constitutional symptoms. Complications include meningoencephalitis and treatment-induced Jarisch-Herxheimer reaction. Diagnosis can be made by microscopic examination of blood smears collected during fever episodes or by molecular methods.

TBRF in international travelers is rare. The GeoSentinel Surveillance Network reported only 4 cases of relapsing fever among 24,920 returning febrile travelers during 1997-2006 (2), and we found only 40 other travel-related cases published since 1982 (Appendix Table 2). Most TBRF infections in travelers are caused by B. crocidurae and almost exclusively acquired in Senegal. Recently, a new species, Candidatus Borrelia kalaharica, was found in 2 travelers to southern Africa $(3,4)$.

Reports on B. persica infections are few and largely restricted to Iran and Israel. Only 2 other cases of $B$. persica infections in travelers returning from Uzbekistan/Tajikistan have been reported $(5,6)$. Considering the wide geographic distribution of the transmitting tick, Ornithodoros tholozani (India, Pakistan, Afghanistan, western China, Kazakhstan, Kyrgyzstan, Tajikistan, Turkmenistan, Uzbekistan, Iran, Iraq, Turkey, Cyprus, Syria, Jordan, Israel, Egypt, and Libya $[7,8])$, considerable underreporting and underrecognition is likely. Although apparently rare, central nervous system involvement and acute respiratory distress syndrome may complicate TBRF caused by B. persica (9).

For patients with periodic fever and supporting exposure risk, clinicians should consider a differential diagnosis of TBRF and carefully examine blood smears by microscopy. Increasingly available PCR and sequencing techniques provide highly sensitive diagnostic tools superior to microscopy.

\section{About the Authors}

Dr. Muigg is a clinician in the Department of Medicine at the Swiss Tropical and Public Health Institute, Basel, Switzerland. Her research interests include infectious diseases with a focus on neglected tropical diseases.

Dr. Seth-Smith is a bioinformatician in the Division of Clinical Bacteriology at the University Hospital, Basel. Her research interests include analyzing bacterial genomes to better understand pathogens, both evolutionarily and within a hospital context.

\section{References}

1. Talagrand-Reboul E, Boyer PH, Bergström S, Vial L, Boulanger N. Relapsing fevers: neglected tick-borne diseases. Front Cell Infect Microbiol. 2018;8:98. https:/ / doi.org/10.3389/fcimb.2018.00098

2. Wilson ME, Weld LH, Boggild A, Keystone JS, Kain KC, von Sonnenburg F, et al.; GeoSentinel Surveillance Network. Fever in returned travelers: results from the GeoSentinel Surveillance Network. Clin Infect Dis. 2007;44:1560-8. https://doi.org/10.1086/518173

3. Fingerle V, Pritsch M, Wächtler M, Margos G, Ruske S, Jung J, et al. "Candidatus Borrelia kalaharica" detected from a febrile traveller returning to Germany from vacation in southern Africa. PLoS Negl Trop Dis. 2016;10:e0004559. https://doi.org/10.1371/journal.pntd.0004559

4. Stete K, Rieg S, Margos G, Häcker G, Wagner D, Kern WV, et al. Case report and genetic sequence analysis of Candidatus Borrelia kalaharica, southern Africa. Emerg Infect Dis. 2018;24:1659-64. https://doi.org/10.3201/ eid2409.171381

5. Kutsuna S, Kawabata H, Kasahara K, Takano A, Mikasa K. The first case of imported relapsing fever in Japan. Am J Trop Med Hyg. 2013;89:460-1. https:/ / doi.org/10.4269/ ajtmh.13-0187 
6. Colin de Verdiere N, Hamane S, Assous MV, Sertour N, Ferquel E, Cornet M. Tickborne relapsing fever caused by Borrelia persica, Uzbekistan and Tajikistan. Emerg Infect Dis. 2011;17:1325-7. 10.3201/eid1707.101894 https://doi.org/10.3201/eid1707.101894

7. Assous MV, Wilamowski A. Relapsing fever borreliosis in Eurasia - forgotten, but certainly not gone! Clin Microbiol Infect. 2009;15:407-14. https://doi.org/10.1111/ j.1469-0691.2009.02767.x

8. Parola P, Raoult D. Ticks and tickborne bacterial diseases in humans: an emerging infectious threat. Clin Infect Dis. 2001;32:897-928. https://doi.org/10.1086/319347

9. Yossepowitch $\mathrm{O}$, Gottesman $\mathrm{T}$, Schwartz-Harari $\mathrm{O}$, Soroksky A, Dan M. Aseptic meningitis and adult respiratory distress syndrome caused by Borrelia persica. Infection. 2012;40:695-7. https://doi.org/10.1007/s15010-012-0296-8

Address for correspondence: Veronika Muigg, Department of Medicine, Swiss Tropical and Public Health Institute, Socinstrasse 57, Basel 4051, Switzerland; email: veronika.muigg@swisstph.ch

\section{Imported Human Babesiosis, Singapore, 2018}

\author{
Poh-Lian Lim, Jean-Marc Chavatte, Shawn Vasoo, \\ Jonathan Yang
}

Author affiliations: Tan Tock Seng Hospital, Singapore (P.-L. Lim, S. Vasoo); Nanyang Technological University, Singapore (P.-L. Lim, S. Vasoo); National Centre for Infectious Diseases, Singapore (J.-M. Chavatte); Eton College, Windsor, UK (J. Yang)

DOI: https://doi.org/10.3201/eid2604.200025

In 2018, Babesia microti infection was diagnosed for a 37-year-old man in Singapore who acquired the infection in the United States. This case highlights the recent rise of tickborne infections in the United States and the risk for their spread, because of increasing global interconnectivity, to regions where they are not endemic.

$B$ abesia spp. are intra-erythrocytic protozoal organisms that can infect mammals and birds. Human babesiosis is an emerging tickborne zoonosis, caused mainly by Babesia microti and transmitted by ixodid ticks. It is endemic to the United States (1-3) and, to a lesser extent, China $(3,4)$. Recently, sporadic cases of human babesiosis caused by several species of Babesia have been reported in other countries: B. microti (Germany, Australia, South Korea), B. microti-like (Japan, Taiwan, China), B. duncani (United States, Canada), B. divergens (Europe), B. venatorum (Europe, China), B. crassa-like (China), B. motasi-like (South Korea), and other cases elsewhere (1-4).

In humans, babesiosis can cause mild influenza-like signs and symptoms, but it can also cause hemolytic anemia and severe infections, especially in asplenic or immunocompromised persons $(1,3)$. Cases of congenital and transfusion-related transmission have been reported (1-4). Since 2011-2015, babesiosis incidence in the United States has risen $(2,5)$. Travel-related tickborne infections in general (6) and cases acquired from North America have been reported $(3,6,7)$. To our knowledge, no case of human babesiosis has been reported in Singapore, but cases of Babesia infection in canids and birds have been recorded (8), suggesting presence of potentially receptive ticks.

On July 23, 2018, a 37-year-old man from the United States sought care at Tan Tock Seng Hospital, Singapore, reporting fever and other influenza-like signs and symptoms that had started on July 5 . The patient had resided in Singapore since 2012, working as a finance professional, but he had traveled to multiple places in the year before his illness. In 2017, he vacationed in Vietnam (Ho Chi Minh City, Danang), Thailand (Bangkok, Pattaya), Indonesia (Lombok, Anambas Islands), and Cambodia (Phnom Penh), all without having received pretravel typhoid vaccine or malaria prophylaxis. In 2018, he traveled to Indonesia (Bali) in January and March, then to the United States during June 14-25, where he visited friends and relatives in Boston (MA), Nantucket (MA), and New York (NY).

The patient did not recall any tick bites but on June 17 noticed a right ankle papule, which lasted 3 weeks. He sought consultation at a travel clinic because of high fever $\left(104^{\circ} \mathrm{F}\right)$, rigors, and headaches, which had persisted and worsened over 18 days. His fever had not resolved with amoxicillin, which he had started taking a week after symptoms onset. He had no relevant medical history or allergies and was taking no other medication. Physical examination findings were unremarkable, including absence of jaundice, hepatosplenomegaly, or eschars.

Laboratory test results revealed moderate thrombocytopenia and anemia, and malaria blood films revealed trophozoites forming in erythrocytes, suggestive of Babesia. The National Public Health Laboratory in Singapore differentiated 